1350 CENTRAL NERVOUS SYSTEM CANDiDIASIS IN AN

INTENSIVE CARE NURSERY (ICN). R.G. Faix (Spon. by G. Wilson), Dept. of Pediatrics, Univ. of Michigan, Ann

Arbor, Mr

Intravenous Amphotericin B (IV AmB) is standard therapy for systemic candidiasis, but penetrates the central nervous system (CNS) poorly. From 1971-83, 22 infants in this ICN developed systemic candidiasis, defined by clinical signs of sepsis, blood and/or CSF cultures (19) or deep tissue histologic specimens positive for Candida (3), and failure to improve without antifungal therapy. Seven infants (31\%) died of this infection. Fourteen of these 22 infants (64\%) had culture or autopsy-proven evidence of Candida CNS infection - 10 had positive CSF cultures; 4 had cerebral or intramedullary cord abscesses at autopsy. Six of these 14 infants $(43 \%)$ with CNS infection died - none of these Six of these 14 infants $(43 \%)$ with CNS infection died - none of these
6 had received systemic antifungal therapy (culture results unavailable 6 had received systemic antifungal therapy (culture results unavailable
before death in 2 cases, isolate wrongly considered transient or contaminant in 3 cases, negative blood and CSF cultures in 1 case). Among the 8 treated survivors of CNS candidiasis, CSF was sterilized with IV AmB alone in only 2 cases. Four others required the addition of flucytosine (FC) which penetrates the CNS well, after $5,7,11$ and 13 days respectively of IV AmB failed to sterilize CSF; one required intraventricular instillation of $\mathrm{AmB}$ after an unsuccessful 21 day course
of IV AmB; one required FC only for CSF sterilization. These of IV AmB; one required FC only for CSF sterilization. These involves the CNS; 2) blood/CSF cultures positive for Candida should be considered significant, until proven otherwise; 3) IV AmB alone is of ten inadequate to sterilize infected CSF and changes in medication or route of administration may be necessary.

\title{
SURVIVAl of Cytomegalovirus (CMV) ON
}

ENVIRONMENTAL SURFACES, R.G. Faix (Spon. by G.

Wilson), Dept. of Peds., Univ. of Michigan, Ann Arbor, MI.

Transmission of CMV from infected newborns to nursery personnel, other adults and other newborns can occur. The role of fomites in this ther aduls and other fresh urine specimens were obtained from 5 patients $(1-5)$ with congenital and 1 patient (6) with acquired CMV infection. Urine in each urine specimen was quantitated in quadriplicate by plaque assay using a methylcellulose overlay technique. Seven $0.2 \mathrm{ml}$ aliquots of each urine specimen were placed on plexiglass that had been cleaned 15 minutes earlier with $0.07 \%$ quaternary ammonium chloride and $70 \%$ isopropyl locis alcohol (standard incubator were maintained at room temperature $\left(25-27^{\circ} \mathrm{C}\right)$ in room air. Separate aliquots were sampled with a moist cotton swab at $1,2,4,8,12,24$ and 48 hours and cultured in duplicate on human foreskin fibroblasts.

$\log _{10}$ plaque-forming units per ml urine Latest CMV recoveryplexiglass (hours) Latest CMV recovery-

blanket (hours)

Live CMV may survive for several hours on nonabsorptive surfaces and at least one hour on absorptive surfaces. Pretreatment of plexiglass with two common nursery disinfectants still permitted viral recovery. Contaminated environmental surfaces may serve as potential reservoirs for nosocomial transmission of CMV. $\dagger 1352$ HYPERTENSION REDEFINED FOR CRITICALIY ILL NEONATES .K.Stork, W.A.Car1o, R.M.Kliegman, A.A. Fanaroff. The diagnosis of neonatal hypertension(HTN) has been complicated by the limited available normative data and hence, arbitrary assignment of the upper limit of normal blood pressure(BP). Our objective was to determine a normative data base. BPs were recorded every $8 \mathrm{hrs}$ for up to 30 days in 121 preterm and 61 term infants who required an umbilical artery catheter(UAC). Dinamap was used after discontinuation of UAC. Infants were grouped as follows: $<1.0 \mathrm{~kg}(n=17), 1.0-1.5 \mathrm{~kg} \quad(n=41), 1.5-2.5$ $\mathrm{kg}(n=63)$ and $>2.5 \mathrm{~kg}(n=61)$. In each group, mean arterial pressure(MAP) rose steeply over the first 5 days. At all times, BP was higher in infants with the greater birth weight(BW). Predictive equations for MAP as a function of postnatal age(PNA) best fit a curvilinear relationship $(M A P=a+b \cdot \ln [$ day]). The following tabulates the MAP ( $\mathrm{mmHg}$ ) and $95 \%$ confidence limits for single measurements.

\begin{tabular}{|c|c|c|c|c|}
\hline & & $5 \mathrm{Kg}$ & $1.5-2.5 \mathrm{Kg}$ & $\mathrm{Kg}$ \\
\hline & & & & $\overrightarrow{4 \varepsilon}$ \\
\hline & $41.4=$ & $47.2 \pm 18.2$ & $50.4 \pm 19.6$ & 7 \\
\hline & 44.6 & $50.1 \pm 18.2$ & $3.2 \pm 19.6$ & \\
\hline
\end{tabular}

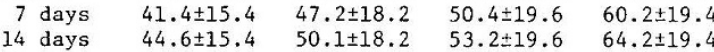

28 days $\quad 47.6 \pm 15.4 \quad 53.0 \pm 18.2 \quad 56.1 \pm 19.6 \quad 68.3 \pm 19.4$ Both PNA and BW markedly influence BP values. By prior definition(MAP $>70 \times 3$ days) $10 \%$ of these asymptomatic neonates would be hypertensive. The present criteria reduces the incidence to $2 \%$ which is in accordance with our previous report of symptomatic HTN. This dynamic definition of the range of BP with respect to $\mathrm{BW}$ and PNA more precisely identifies HTN in sick neonates.
NEONATAL HYPERTENSION APPEARS UNRELATED TO AORTIC CATHETER POSITION. E.K.Stork, W.A.Carlo, R.M. Kliegman, A.A.Fanaroff, CWRU, Dept.Peds., Cleve, OH In 1981 we reported severe symptomatic hypertension (HTN) in $2 \%$ of al1 neonatal admissions, $85 \%$ with umbilical arterial catheters(UAC) above the renal arteries. To prospectively evaluate the occurrence of HTN with respect to catheter tip position, we randomly placed high (T6-10) vs low (L3-5) UACs. This preliminary report reviews the first 182 infants analyzed. Birth weight (BW) ranged from $0.72-4.4 \mathrm{Kg}$, mean $2.0 \mathrm{Kg}$, with gestational age (GA) 24-44 wks, mean 32 wks. BP was recorded every 8 hours via the UAC (68\% Orange Medical Instruments [OMI] Continuous Recording Catheters, $32 \%$ Argyle Catheters) or Dinamap for a maximum of 30 days. Problems with catheter insertion occurred in $39 / 182(21 \%)$ without regard to catheter type: $28 / 124(22.6 \%)$ OMI, $11 / 85(18.9 \%)$ Argyle, $p>.10$. BP showed no diurnal variation but was related to $\mathrm{BW}, \mathrm{GA}$ and postnatal age. HTN(mean arterial pressure $\geqslant 70 \times 3$ days) occurred in $19 / 182(10 \%)$.

$\begin{array}{lcccc} & \text { High UAC } & \text { LOW UAC } & & \\ \text { Hypertension } & 7 / 85(8 \%) & 12 / 97(12 \%) & \text { NS } & p>.10 \\ \text { + Leg Perfusion } & 12 / 85(14 \%) & 25 / 97(26 \%) & & p<.05 \\ \text { NEC } & 5 / 85(6 \%) & 3 / 97(3 \%) & \text { NS } & p>.10\end{array}$
In al1 cases HTN was asymptomatic and did not warrant treatment. Compromised perfusion to legs(unilateral cyanosis, pallor, los of pulses) was greater with low UACs; other complications were unrelated to catheter tip position. We conclude that HTN bears no relationship to catheter position; the prevailing numerical definition over-diagnoses HTN in high risk neonates.
1354 ULTRASONIC IMAGING OF BRAIN PARENCHYMA. Jason C. Bir Iica1 College, Dept. of Radiology and Northwestern I11inois.

Ultrasonic imaging has not been used for primary visualization of brain parenchyma in fetuses or neonates, although it is applied routinely for evaluation of ventricles (and other fluid collections) and for the detection of intracranial hemorrhage an neoplasms. We have begun investigating cerebal parenchymal appearance directly with a large aperture, dynamically focused array immaging system having improved signal to noise ratio and regional magnification features.

We find that bone is not an absolute deterant to imaging, through 6 weeks post term, a1though some refraction effects are noted when molded heads (i.e. surviving small premature infants) are viewed in coronal planes through a water path. Convolutional markings in the cengulate region in midsagittal views in fetuses and in newborn infants show a progression in complexity between 28 and 32 weeks and after 37 weeks in accord with the necropsy correclation with gestational age. The definition of markings and primary reflectivity increases progressively in the third trimester. An Increased reflectivity and damped anterior cerebral branch artery pulsations occur after asphyxial episodes and are interpreted as an extracellular f1uid shift. Decreased echodensity and splaying of markings are typical of edema. Thickened sulci herald aophy in subjects beyond 4 months of age.

\section{A CRITICAL EVALUATION OF SERUM DEXTROSTIX (DTX) IN NEWBORNS. Yucel S. Atakent, Usha Gupta,
Ellis N. Beesley, Angelo Ferrara. New York University} Ellis N. Beesley, Angelo Ferrara. New York

To detect \& Rx hypoglycemia (serum $40 \mathrm{mg} / \mathrm{dl}$ ) early, reagent strips $(\mathrm{dtx})$ are used to screen newborns (NB) at risk. If the dtx is low in an asymptomatic infant, the glucose (glc) lab result is obtained before Rx. This is done because of high incidence of false hypoglc Dx by dtx. The aim of this study was to assess accuracy of dtx when effect of hct is eliminated. 14 Blood (bl) samples in $12 \mathrm{NB}(\mathrm{BW} \pm 1 \mathrm{SD}: 2.2 \pm 1.2 \mathrm{~kg}$ ) were
examined. Part of the sample was used for dtx (read at 60") \& hct examined. Part of the sample was used for dtx (read at 60") \& hct
determination (dtern). After centrifugation the remaining serum (sm)
sample was used for sample was used for lab glc dtern (Hexokinase) \& for repeated sm dtx read at $45 ", 50 ", 55^{\prime \prime} \& 60^{\prime \prime}$. Thus a total of $5 \mathrm{dtx}$ readings ( 1 whole bl \& $4 \mathrm{sm}$ ) with a calibrated glucometer were compared to lab result for each patient. Results: no one had hypoglc as determined by the lab

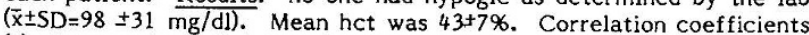
$(r)$ of regressions of dtx readings on lab glc dterns for each time period were statistically significant $(\mathrm{p}<.001)$. The $r$ 's for all time periods for were $s$ tatistically significant $(p<.001)$. The $r$ 's for all time periods for
sera were $>$ for whole bl. When paired comparisons were made between "r's" of each time period for sm \& whole bl, only the "r" of the $50 "(.98)$ for sm was significantly different from whole bl $(.91)(P<.025)$. DTX read with $\mathrm{sm}$ at 50 " also showed smaller differences to lab glc dtern compared to whole bl $(9 \pm 7$ vs $25 \pm 14 \mathrm{mg} \%, \mathrm{p}<0.025)$. Only 1 of $14 \mathrm{dtx}$ measurements each with $\mathrm{sm}$ at $50^{\prime \prime} \&$ whole bl were falsely high compared to lab dterns. In the event of a low dtx reading during may facilitate Dx \& Rx of hypoglc long before lab results are available. 